\title{
INTEGRAÇÃO DE SENSORES GEOFÍSICOS E GEOESTATÍSTICA PARA MAPEAR ATRIBUTOS DO SOLO
}

\author{
Hugo M Rodrigues $^{(\mathrm{a})}$, Gustavo M Vasques ${ }^{(\mathrm{b})}$ \\ (a) Geógrafo, Universidade Federal Fluminense, hugomr@id.uff.br \\ (b) Pesquisador, Embrapa Solos, gustavo.vasques@embrapa.br
}

\section{Eixo: SOLOS E PAISAGENS}

\begin{abstract}
Resumo
O conhecimento da variação espacial dos atributos do solo é primordial para o gerenciamento do sistema agrícola. O objetivo do trabalho foi mapear, em uma área de 3,4 ha em Seropédica, RJ, a condutividade elétrica aparente, susceptibilidade magnética e os teores dos elementos radioativos tório e urânio do solo, medidos por sensores geofísicos in situ, e os teores de argila, ferro, carbono orgânico e umidade, e capacidade de troca catiônica do solo, medidos em laboratório, em 130 pontos amostrais. Comparou-se krigagem ordinária com krigagem universal utilizando as coordenadas geográficas x e y e a elevação como covariáveis. Os dois métodos de krigagem produziram mapas com padrão de distribuição espacial e índices de incerteza semelhantes. Outrossim, os padrões de dependência e distribuição espacial foram similares entre os atributos geofísicos e os de laboratório, evidenciando o potencial da geofísica para o mapeamento de atributos do solo.
\end{abstract}

Palavras chave: Sensoriamento proximal do solo, Pedometria, Geoestatística

\section{Introdução}

As informações obtidas por meio do mapeamento da variação dos atributos do solo são utilizadas como subsídio para o planejamento das práticas de manejo para a conservação do solo e da água (Mello et al., 2006; Silva et al., 2008), produção de culturas agrícolas (Sanchez et al., 2005; Mann et al., 2010), estudos relacionados à gênese (Camargo et al., 2008), delineamento amostral (Montanari et al., 2005), estudos de emissão de $\mathrm{CO}_{2}$ (Panosso et al., 2009; Brito et al., 2010) e outros.

A geoestatística se constitui em um conjunto de conceitos e ferramentas utilizados para a caracterização da variação espacial e/ou temporal de grandezas ou fenômenos que manifestam autocorrelação espacial e/ou temporal, respectivamente. Considerando o espaço, a geoestatística baseia-se no pressuposto de que observações localizadas mais próximas entre si são mais parecidas do que as que se localizam mais distantes. Esse comportamento atesta a existência de dependência espacial, a qual pode ser expressa por meio da análise de semivariograma da variável de interesse. A análise do semivariograma, além de permitir a caracterização da estrutura de dependência espacial, é um passo fundamental no processo de interpolação de valores de lugares amostrados para lugares não amostrados por krigagem para a geração de mapas da distribuição espacial da variável de interesse. 
Esse trabalho parte dos resultados obtidos na mesma área de estudo por Rodrigues; Vasques (2016) e Rodrigues et al. (2016), que encontraram relação entre a condutividade elétrica aparente (CEa), a susceptibilidade magnética (SM) e a distribuição dos elementos radioativos tório (Th) e urânio (U) do solo, medidos por sensores geofísicos no campo, e atributos do solo amostrados e medidos utilizando métodos convencionais de coleta e análise em laboratório, sendo eles os teores de argila, ferro $(\mathrm{Fe})$, carbono orgânico (CO) e umidade, e capacidade de troca catiônica (CTC). Esses estudos utilizaram krigagem ordinária para interpolar os valores de cada atributo, separadamente, e observaram que os atributos do solo medidos em laboratório apresentaram padrões de distribuição e dependência espaciais semelhantes entre si e em relação àqueles dos sensores geofísicos, sendo que esses padrões estavam relacionados aos padrões de relevo da área.

Dessa forma, o objetivo do presente trabalho é avaliar a krigagem universal para a interpolação dos mesmos atributos do solo, considerando os padrões geográficos e de relevo como covariáveis, se partindo do pressuposto de que essas covariáveis melhoram a qualidade dos mapas de atributos produzidos em relação aos mapas de referência dos respectivos atributos produzidos usando krigagem ordinária. Ou seja, se pretende avaliar se a inclusão das coordenadas geográficas x e y e da elevação no modelo de interpolação via krigagem universal melhora a qualidade dos mapas gerados, ou seja, diminui os seus erros de predição (interpolação).

\section{Materiais e Métodos}

Esse estudo foi conduzido no município de Seropédica, RJ, em um campo experimental da Empresa de Pesquisa Agropecuária do Estado do Rio de Janeiro (PESAGRO) (Figura 1). A área de estudo possui 3,4 ha e encontra-se cultivada com capim colonião (Panicum maximum Jacq.) há mais de uma década. Tratase de uma vertente representativa da região, dominada por uma topossequência de Argissolos a Planossolos da parte mais elevada para a mais baixa. A litologia é composta por um embasamento de rochas do Complexo do Litoral Fluminense, em que predominam gnaisses, com intrusões de rochas basálticas e rochas alcalinas, localmente superposto por sedimentos flúvio-lacustres do Quaternário, conhecidos como Formação Piranema (Mello, 1998; Góes, 1994). A região possui clima variando de subtropical a tropical chuvoso, com inverno seco e verão quente e úmido.

Foi estabelecida uma malha amostral de 20 x $20 \mathrm{~m}$, constituída por 7 transectos longitudinais à topossequência com 15 pontos cada um, perfazendo 105 pontos amostrais (Figura 1). Em todos os pontos dessa malha foram medidas a CEa e a SM usando o sensor KT-10 S/C (Terraplus Inc., Richmond Hill, Canadá) e os teores de Th e U equivalentes (eTh e eU, respectivamente) usando o sensor RS-230 BGO (Radiation Solutions Inc., Mississauga, Canadá). As medições foram tomadas encostando os sensores 
portáteis na superfície do solo, em um local liso e plano, após a retirada da serapilheira. No caso do sensor KT-10 S/C, a medição se dá por meio de indução eletromagnética: uma bobina transmissora gera campos magnéticos primários, os quais induzem uma corrente elétrica no solo, que por sua vez cria um campo magnético secundário; uma bobina receptora calcula a magnitude da fração desse campo secundário que retorna à segunda bobina, que soma e amplifica esses sinais retornando uma voltagem que se relaciona à CEa ponderada em função da profundidade (CORWIN; LESCH, 2005). A profundidade de leitura depende da frequência, da distância entre as bobinas de transmissão e recepção e do modo de operação, seja ele em dipolo horizontal ou vertical. O sensor RS-230 BGO, por sua vez, capta a contagem de raios gama emitidos da superfície do solo e separa essa contagem total em função da energia diagnóstica de cada elemento (U e Th), permitindo estimar a concentração destes, sendo que as concentrações são calculadas na forma de elementos equivalentes, pois o sensor não mede diretamente a concentração dos elementos (WILFORD et al. 1997). As medições do sensor RS-230 BGO foram obtidas com leituras de 120 segundos.

Estabeleceu-se uma malha amostral de $10 \times 10 \mathrm{~m}$ (377 pontos) objetivando alocar 25 pontos usando delineamento amostral por hipercubo latino condicionado (cLHS) (MINASNY; MCBRATNEY, 2006) para avaliação dos erros de predição dos atributos do solo, ou seja, da qualidade dos mapas, por meio de validação independente. Nos 130 pontos alocados (105 pontos na malha de 20 x $20 \mathrm{~m}$ mais 25 pontos distribuídos por cLHS), foram abertas minitrincheiras onde foram coletadas amostras deformadas de solo em 0-10 cm para medição em laboratório dos teores de argila, Fe e CO, e da CTC, bem como amostras indeformadas usando anel volumétrico de $100 \mathrm{~cm}^{3}$ para medição do teor de umidade volumétrica.

Em laboratório, foram medidos, segundo Embrapa Solos (2011), o teor de argila pelo método do densímetro, o teor de $\mathrm{CO}$ por meio da oxidação da matéria orgânica por via úmida com dicromato de potássio em meio sulfúrico e o teor de Fe após extração usando ácido sulfúrico. Para o cálculo da CTC, foram somados os teores dos cátions trocáveis cálcio e magnésio, extraídos por cloreto de potássio, potássio e sódio, extraídos por solução de ácido clorídrico mais ácido sulfúrico, e hidrogênio e alumínio, extraídos por acetato de cálcio.

A análise exploratória dos dados incluiu a descrição estatística dos atributos do, seguida da análise de correlação linear entre os atributos do solo e covariáveis geográficas. Para modelagem da dependência espacial e posterior interpolação dos dados, foram utilizados as 105 observações na malha de 20 x $20 \mathrm{~m}$. Foi utilizado o modelo de semivariograma esférico, cujos parâmetros foram ajustados pelo método empírico (opção method=7) da função fit.variogram do pacote gstat (PEBESMA, 2004) do 
XVII Simpósio Brasileiro de Geografia Fisica Aplicada

I Congresso Nacional de Geografia Física

\section{OS DESAFIOS DA GEOGRAFIA FÍSICA NA FRONTEIRA DO CONHECIMENTO \\ Instituto de Geociências - Unicamp \\ Campinas - SP \\ 28 de Junho à 02 de Julho de 2017}

programa R (R CORE TEAM, 2015), ou manualmente quando necessário. Usou-se transformação para logaritmo nas variáveis Fe e SM para aproximarem distribuição de frequência normal.

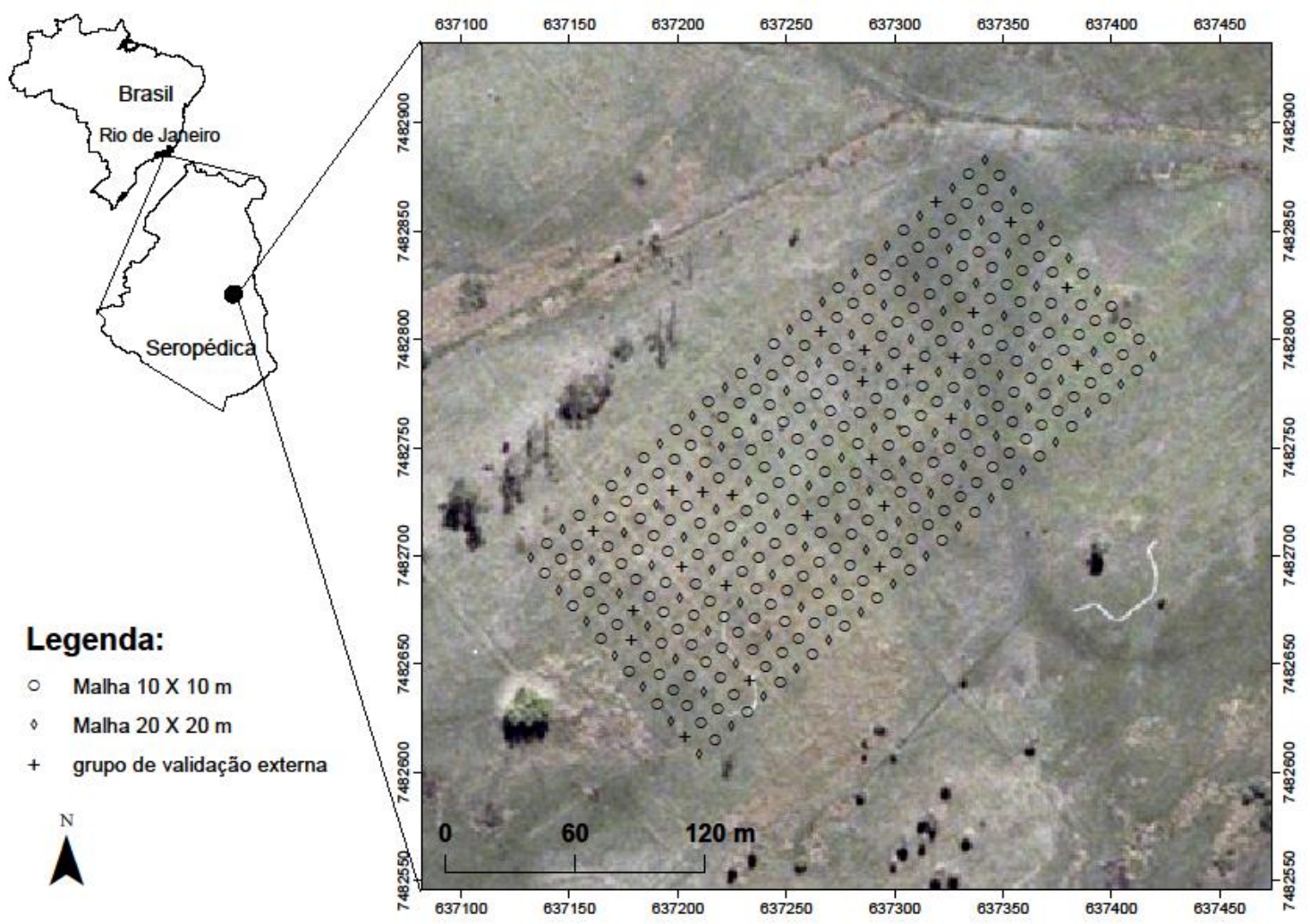

Figura 1 - Mapa de localização da área de estudo e delineamento amostral. A malha de 20 x $20 \mathrm{~m}$ e os 25 pontos de validação externa estão dispostos sobre a malha de 10 x 10 m. Projeção WGS84 UTM 23S.

Utilizou-se krigagem ordinária e krigagem universal (WEBSTER; OLIVER, 2007) para interpolação dos dados e produção dos mapas dos atributos medidos, utilizando como variáveis dependentes os atributos do solo, respectivamente, e como variáveis independentes, ou covariáveis, as coordenadas geográficas x e y e a elevação, sendo todos os mapas interpolados na resolução espacial de $1 \mathrm{~m}$. Para krigagem ordinária, foram usadas as funções krige, para dados na unidade original, e krigeTG, para os dados transformados, ambas do pacote gstat. A função krigeTG realiza krigagem do tipo lognormal, reconvertendo os valores estimados para a unidade original após a krigagem ordinária na unidade logarítmica (PEBESMA, 2004). Para a krigagem universal, foi usada a função krige, nesse caso reconvertendo-se manualmente via transformação exponencial os valores interpolados em logaritmo para as unidades originais a fim de produzir o mapa final e realizar a validação externa. 


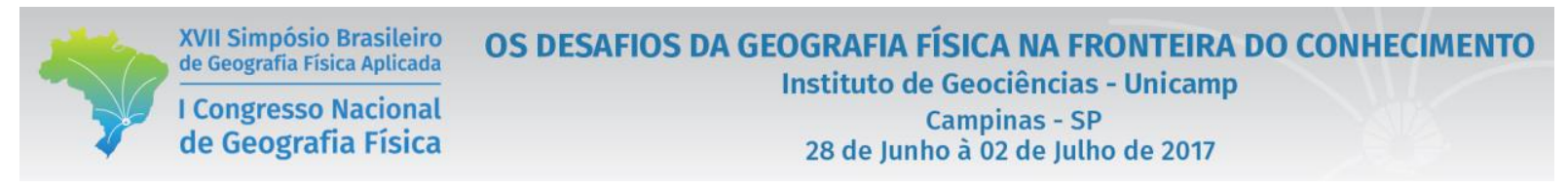

Os 25 pontos alocados por cLHS foram usados exclusivamente para o cálculo dos erros de predição dos atributos do solo, sendo eles o erro médio (EM) e a raiz do erro quadrado médio (REQM), os quais foram usados para comparar a qualidade dos mapas gerados pelos dois métodos de interpolação.

\section{Resultados e Discussão}

As estatísticas descritivas dos atributos do solo encontram-se na Tabela I, enquanto as correlações entre os atributos do solo e coordenadas geográficas e elevação são apresentadas na Tabela II. As maiores correlações foram observadas entre a argila e a elevação $(0,71)$ e o $\log (\mathrm{Fe})$ e a elevação $(0,64)$. Entre as variáveis obtidas por sensores geofísicos, o eTh foi a que obteve as maiores correlações com as coordenadas e elevação. Essas correlações indicam o potencial da krigagem universal de produzir mapas de atributos do solo que considerem os padrões de distribuição espacial das coordenadas geográficas e elevação, já que as mesmas entram nos modelos de interpolação como covariáveis. Ou seja, elas representam na krigagem universal o modelo de tendência espacial global dos atributos do solo, enquanto a krigagem dos resíduos desse modelo representa a tendência espacial local dos atributos (WEBSTER; OLIVER, 2007).

Tabela I - Estatísticas descritivas das variáveis do solo.

\begin{tabular}{lccccccc}
\hline Variável & Obs. & Mínimo & Máximo & Média & Mediana & Desv. pad. & Assim. \\
\hline Argila $\left(\mathrm{g} \mathrm{kg}^{-1}\right)$ & 129 & 3,7 & 28,0 & 11,3 & 11,4 & 3,9 & 0,65 \\
Fe $\left(\mathrm{g} \mathrm{kg}^{-1}\right)$ & 129 & 20,0 & 380,0 & 175,0 & 160,0 & 93,4 & 0,25 \\
$\mathrm{CO}\left(\mathrm{g} \mathrm{kg}^{-1}\right)$ & 129 & 10,0 & 84,0 & 28,6 & 26,0 & 14,4 & 1,35 \\
$\mathrm{CTC}\left(\mathrm{cmol}_{\mathrm{c}} \mathrm{kg}^{-1}\right)$ & 129 & 2,6 & 12,9 & 6,9 & 6,9 & 2,0 & 0,24 \\
Umid $\left(\% \mathrm{v} \mathrm{v}^{-1}\right)$ & 129 & 4,2 & 31,7 & 15,3 & 15,6 & 5,6 & 0,27 \\
$\mathrm{SM}\left(10^{-3} \mathrm{SI}\right)$ & 130 & 0,02 & 3,8 & 0,35 & 0,18 & 0,45 & 4,23 \\
$\mathrm{CEa}\left(\mathrm{S} \mathrm{m}{ }^{-1}\right)$ & 130 & 0 & 4,9 & 1,19 & 0,8 & 1,09 & 1,18 \\
eTh $(\mathrm{ppm})$ & 130 & 0 & 13,8 & 6,30 & 5,8 & 2,76 & 0,44 \\
eU $(\mathrm{ppm})$ & 130 & 0,5 & 3,4 & 1,27 & 1,2 & 0,43 & 1,32 \\
\hline
\end{tabular}

Obs., número de observações; Desv. pad., desvio padrão; Assim., coeficiente de assimetria; Fe, teor de ferro; CO, teor de carbono orgânico; CTC, capacidade de troca catiônica; Umid, umidade volumétrica; SM, susceptibilidade magnética; $\mathrm{CEa}$, condutividade elétrica aparente; eTh, teor de tório equivalente; eU, teor de urânio equivalente.

Por outro lado, o teor de eU mostrou correlação não significativa com todas as variáveis, além de padrões espaciais contrastantes. Esse comportamento distinto pode ser reflexo da alta reatividade e mobilidade do U no ambiente e/ou da sua baixa interação com as outras variáveis do solo. 
Tabela II - Índices de correlação linear entre as variáveis do solo e as coordenadas geográficas e elevação.

\begin{tabular}{cccc}
\hline Variável & $\mathrm{X}$ & $\mathrm{Y}$ & Elevação \\
\hline Argila & $-0,63^{*}$ & $-0,56^{*}$ & $0,71^{*}$ \\
$\log (\mathrm{Fe})$ & $-0,45^{*}$ & $-0,60^{*}$ & $0,64^{*}$ \\
$\mathrm{CO}$ & $-0,33^{*}$ & $-0,28^{*}$ & $0,31^{*}$ \\
$\mathrm{CTC}$ & $-0,53^{*}$ & $-0,48^{*}$ & $0,56^{*}$ \\
Umid & $-0,29^{*}$ & $-0,10 \mathrm{~ns}$ & $0,22^{*}$ \\
$\log (\mathrm{SM})$ & $-0,44^{*}$ & $-0,36^{*}$ & $0,46^{*}$ \\
$\mathrm{CEa}$ & $-0,25^{*}$ & $-0,26^{*}$ & $0,30^{*}$ \\
eTh & $-0,56^{*}$ & $-0,41^{*}$ & $0,57^{*}$ \\
eU & $0,20^{*}$ & $0,21^{*}$ & $-0,19^{*}$ \\
\hline
\end{tabular}

$\log (\mathrm{Fe}), \log$ aritmo natural do teor de ferro; $\mathrm{CO}$, teor de carbono orgânico; CTC, capacidade de troca catiônica; Umid, umidade volumétrica; $\log (\mathrm{SM})$, logaritmo natural da susceptibilidade magnética; $\mathrm{CEa}$, condutividade elétrica aparente; eTh, teor de tório equivalente; eU, teor de urânio equivalente; $\mathrm{X}$, coordenada $\mathrm{x}$; Y, coordenada y. *, correlação significativa ao nível de 0,05 de significância; ns, correlação não significativa.

As estruturas de dependência espacial das variáveis do solo exibiram padrões bem contrastantes entre os métodos de interpolação usados (Tabela III). O patamar diminuiu para todos os atributos do solo, exceto o eU, quando se considerou a krigagem universal, pois as covariáveis coordenadas geográficas e elevação explicaram parte da variância dos atributos do solo. As relações efeito pepita/patamar também aumentaram na krigagem universal, indicando menor continuidade espacial dos atributos após a remoção da tendência global, estruturada, da variância dos atributos em função das covariáveis. Por outro lado, na maioria dos casos, observou-se o aumento da variância não estruturada, aleatória, representada pelo efeito pepita, com a krigagem universal.

Os padrões de distribuição espacial dos atributos do solo gerados pelos dois métodos de interpolação foram muito semelhantes para cada atributo, respectivamente, e esses padrões de distribuição espacial, em geral, se repetiram entre atributos do solo (Figura 2). Ao se utilizar as coordenadas geográficas e elevação como covariáveis, se conseguiu incorporar a influência do relevo na distribuição espacial dos atributos do solo, ainda que os mapas produzidos por krigagem universal sejam muito parecidos com aqueles produzidos por krigagem ordinária, com exceção dos mapas da $\mathrm{CEa}$, que apresentaram diferenças mais marcantes nos padrões produzidos pelos dois métodos. Os índices de incerteza dos mapas gerados confirmam a semelhança entre os mapas do mesmo atributo, pois os dois métodos de interpolação usados produzem mapas com praticamente a mesma qualidade de predição para todos os atributos do solo, respectivamente (Tabela IV). 
XVII Simpósio Brasileiro de Geografia Física Aplicada

I Congresso Nacional de Geografia Física

\section{OS DESAFIOS DA GEOGRAFIA FÍSICA NA FRONTEIRA DO CONHECIMENTO Instituto de Geociências - Unicamp$$
\text { Campinas - SP }
$$ \\ 28 de Junho à 02 de Julho de 2017}

Tabela III - Parâmetros dos semivariogramas das variáveis do solo, considerando-se os dois tipos de krigagem.

\begin{tabular}{lcccc}
\hline Variável & Efeito pepita & Patamar & Efeito pepita/patamar & Alcance $(\mathrm{m})$ \\
\cline { 2 - 5 } Argila & \multicolumn{4}{c}{ Krigagem ordinária } \\
$\log (\mathrm{Fe})$ & 0,02 & 13000 & 0,03 & 261 \\
CO & 7,0 & 0,25 & 0,11 & 181 \\
CTC & 2,40 & 2,08 & 0,56 & 240 \\
Umid & 8,5 & 24,8 & 1,15 & 190 \\
$\log (\mathrm{SM})$ & 0,38 & 0,63 & 0,34 & 208 \\
CEa & 0,78 & 0,55 & 0,60 & 80 \\
eTh & 2,6 & 8,3 & 1,42 & 290 \\
eU & 0,17 & 0,30 & 0,31 & 281 \\
& & & 0,56 & 300 \\
Argila & 1158 & 3691 & 0,31 & 103 \\
$\log (\mathrm{Fe})$ & 0,03 & 0,11 & 0,25 & 84 \\
CO & 7,8 & 6,9 & 1,13 & 164 \\
CTC & 2,46 & 1,22 & 2,02 & 133 \\
Umid & 8,2 & 12,4 & 0,66 & 88 \\
$\log (\mathrm{SM})$ & 0,40 & 0,58 & 0,69 & 72 \\
CEa & 0,60 & 0,38 & 1,58 & 50 \\
eTh & 3,2 & 2,0 & 1,60 & 110 \\
eU & 0,15 & 0,50 & 0,30 & 400 \\
\hline
\end{tabular}

$\log (\mathrm{Fe}), \log a r i t m o$ natural do teor de ferro; CO, teor de carbono orgânico; CTC, capacidade de troca catiônica; Umid, umidade volumétrica; $\log (\mathrm{SM})$, logaritmo natural da susceptibilidade magnética; CEa, condutividade elétrica aparente; eTh, teor de tório equivalente; eU, teor de urânio equivalente.
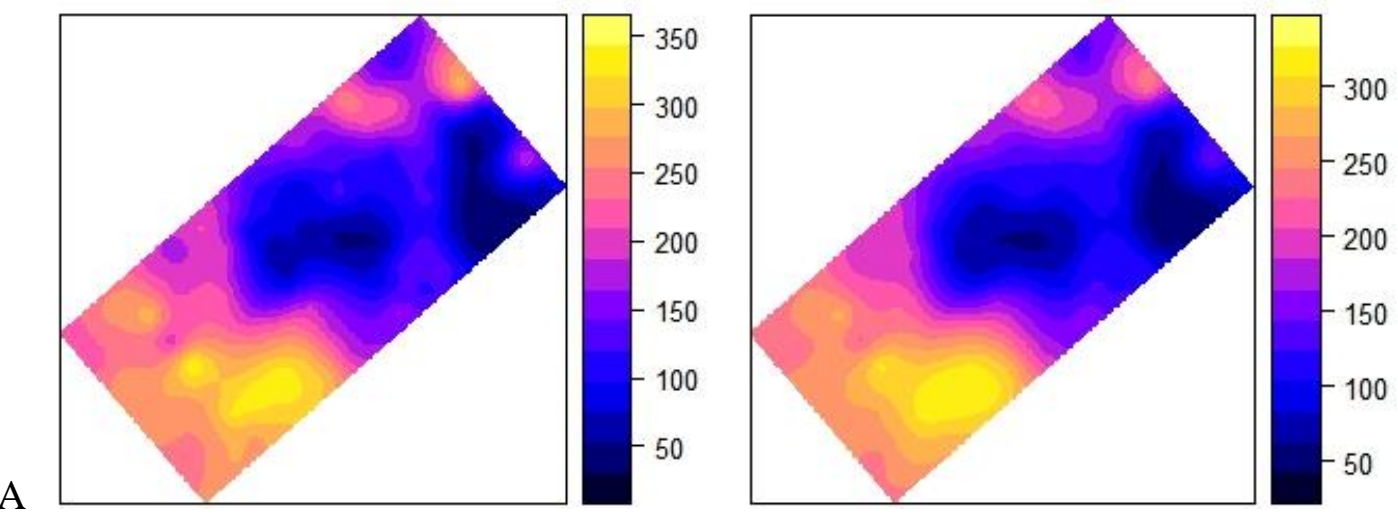
XVII Simpósio Brasileiro de Geografia Fisica Aplicada

I Congresso Nacional de Geografia Física
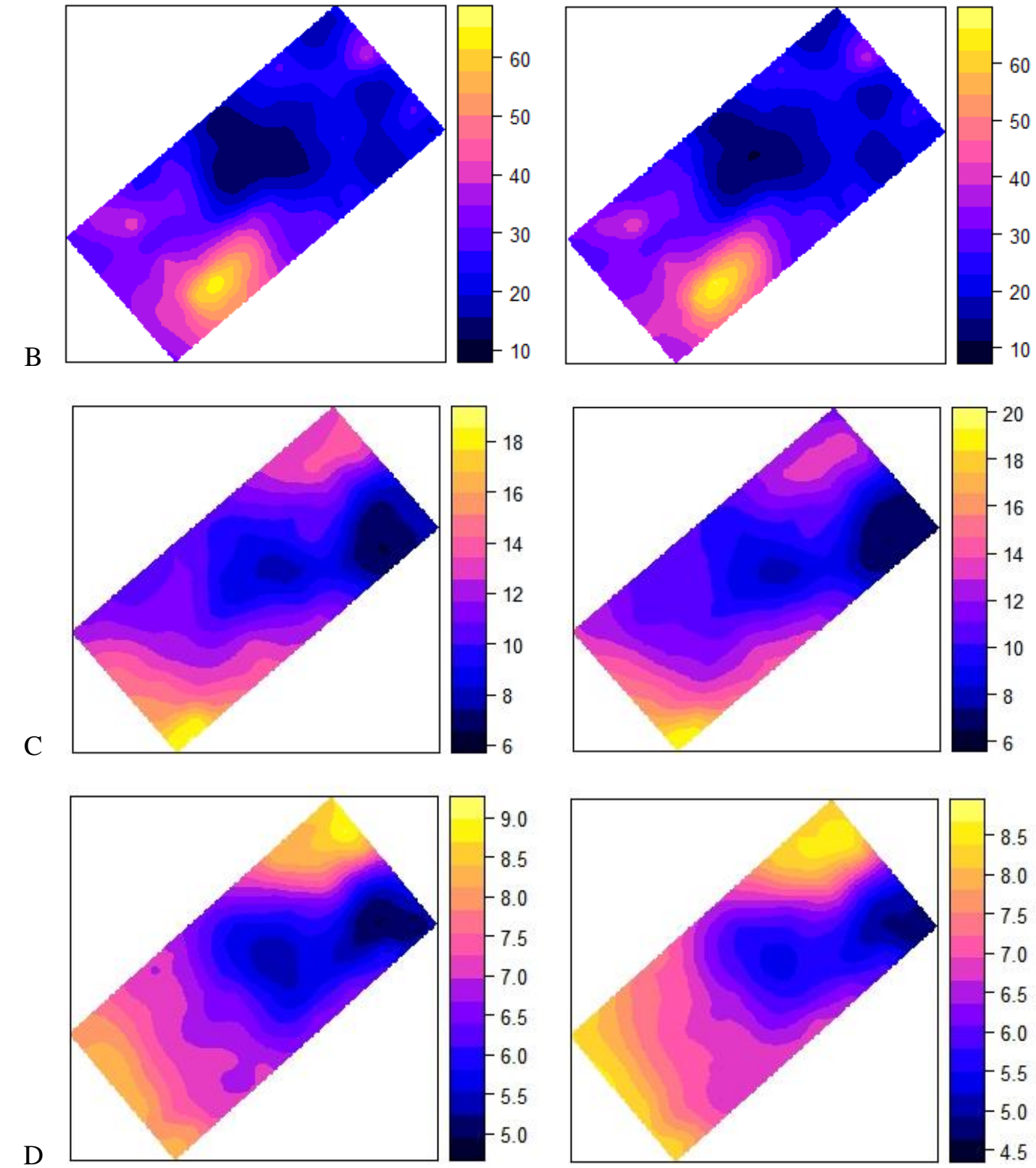
XVII Simpósio Brasileiro de Geografia Fisica Aplicada

I Congresso Nacional de Geografia Física
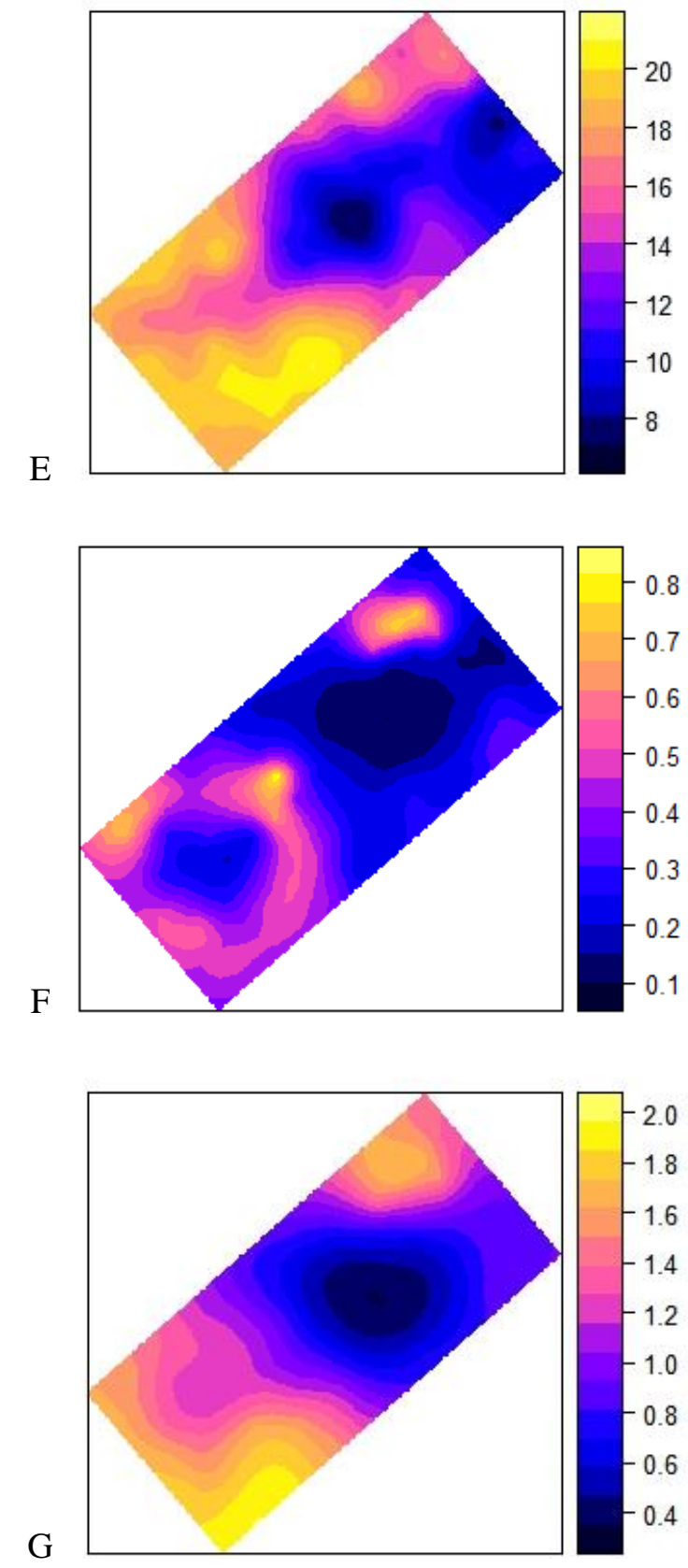

\section{OS DESAFIOS DA GEOGRAFIA FÍSICA NA FRONTEIRA DO CONHECIMENTO Instituto de Geociências - Unicamp Campinas - SP \\ 28 de Junho à 02 de Julho de 2017}
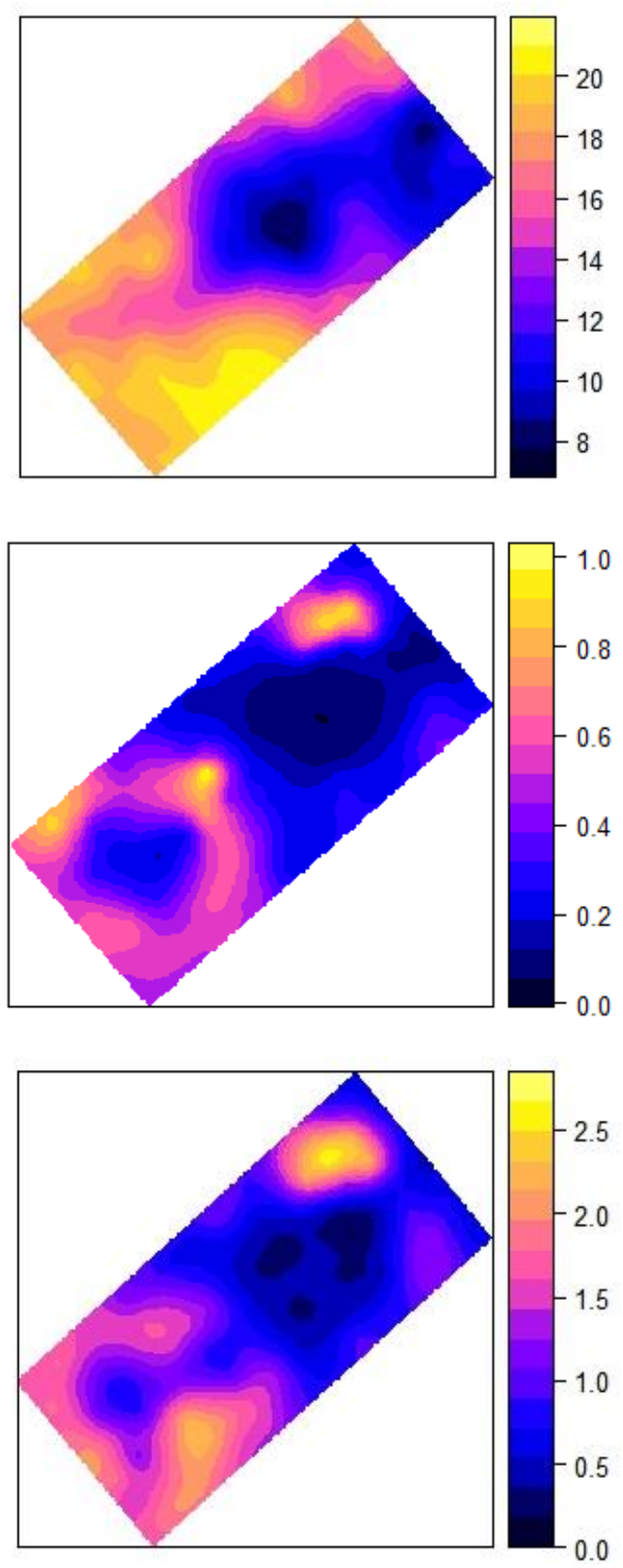

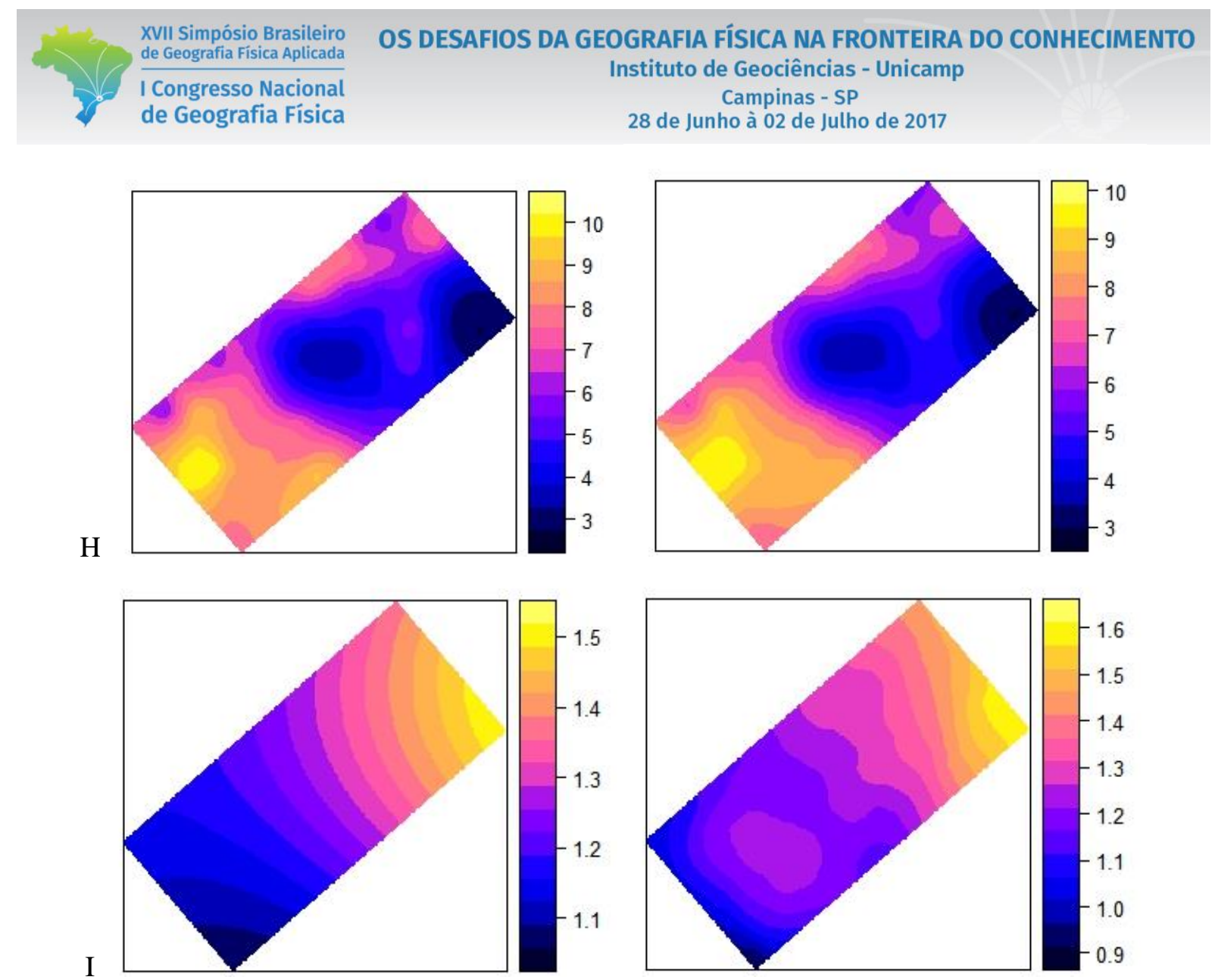

Figura 2 - Mapas dos atributos do solo produzidos por krigagem ordinária (esquerda) e krigagem universal (direita), sendo: (A) teor de argila em 0-10 $\mathrm{cm}\left(\mathrm{g} \mathrm{kg}^{-1}\right)$; (B) teor de ferro em 0-10 cm $\left(\mathrm{g} \mathrm{kg}^{-1}\right)$; teor de carbono orgânico em 0-10 cm $\left(\mathrm{g} \mathrm{kg}^{-1}\right)$; (D) capacidade de troca catiônica em 0-10 $\mathrm{cm}\left(\mathrm{cmol}_{\mathrm{c}} \mathrm{kg}^{-1}\right) ;(\mathrm{E})$ umidade volumétrica em $0-10 \mathrm{~cm}(\%)$; (F) susceptibilidade magnética superficial $\left(10^{-3} \mathrm{SI}\right) ;(\mathrm{G})$ condutividade elétrica aparente superficial $\left(\mathrm{S} \mathrm{m}^{-1}\right)$; $(\mathrm{H})$ teor de tório equivalente superficial $\left(\mathrm{mg} \mathrm{kg}^{-1}\right)$; e (I) teor de urânio equivalente superficial $\left(\mathrm{mg} \mathrm{kg}^{-1}\right)$. 


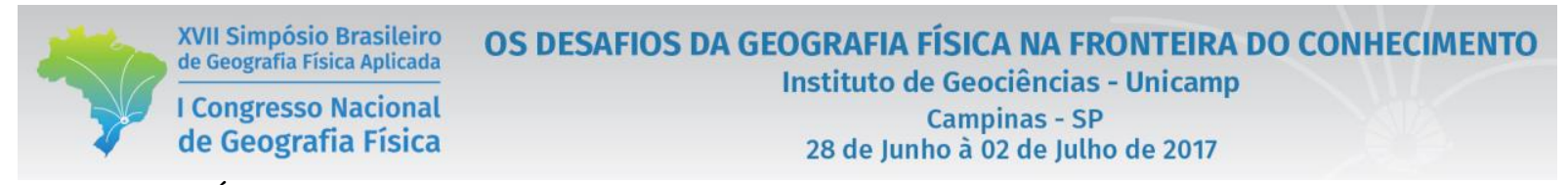

Tabela IV. Índices de incerteza dos mapas obtidos por validação independente usando os 25 pontos alocados por cLHS, considerando-se os dois tipos de krigagem.

\begin{tabular}{lcccc}
\hline \multirow{2}{*}{ Variável } & \multicolumn{2}{c}{ EM } & REQM & \multicolumn{2}{c}{ EM } & REQM \\
\cline { 2 - 5 } & \multicolumn{2}{c}{ Krigagem ordinária } & \multicolumn{2}{c}{ Krigagem universal } \\
\hline Argila $\left(\mathrm{g} \mathrm{kg}^{-1}\right)$ & 21 & 60 & 20 & 61 \\
$\mathrm{Fe}\left(\mathrm{g} \mathrm{kg}^{-1}\right)$ & 7,4 & 14,4 & 7,5 & 14,4 \\
$\mathrm{CO}\left(\mathrm{g} \mathrm{kg}^{-1}\right)$ & 1,2 & 2,9 & 1,3 & 2,9 \\
$\mathrm{CTC}\left(\mathrm{cmol}_{\mathrm{c}} \mathrm{kg}^{-1}\right)$ & 0,87 & 1,53 & 0,87 & 1,52 \\
$\mathrm{Umid}\left(\% \mathrm{v} \mathrm{v}^{-1}\right)$ & 4,2 & 5,8 & 4,2 & 5,9 \\
$\mathrm{SM}\left(10^{-3} \mathrm{SI}\right)$ & 0,05 & 0,34 & 0,02 & 0,33 \\
$\mathrm{CEa}(\mathrm{S} \mathrm{m}$ & -1 & 1,26 & 0,53 & 1,19 \\
eTh $(\mathrm{ppm})$ & 0,52 & 1,7 & 0,6 & 1,8 \\
$\mathrm{eU}(\mathrm{ppm})$ & 0,5 & 0,32 & 0,06 & 0,34 \\
\hline
\end{tabular}

EM, erro médio; REQM, raiz do erro quadrado médio; Fe, teor de ferro; $\mathrm{CO}$, teor de carbono orgânico; CTC, capacidade de troca catiônica; Umid, umidade volumétrica; SM, susceptibilidade magnética; CEa, condutividade elétrica aparente; eTh, teor de tório equivalente; eU, teor de urânio equivalente.

\section{Conclusões}

A krigagem ordinária e a krigagem universal produziram mapas muito semelhantes dos atributos do solo, respectivamente, inclusive com índices de incerteza praticamente iguais, onde se conclui que os valores observados dos diferentes atributos do solo trazem consigo, implicitamente, a influência das coordenadas geográficas x e y e da elevação nos seus padrões espaciais;

Os dois sensores geofísicos utilizados (KT-10 S/C e RS-230 BGO) apresentam potencial para estimar o padrões de dependência e distribuição espacial dos teores de argila, ferro, carbono orgânico e umidade volumétrica, e capacidade de troca catiônica do solo.

\section{Referências}

BRITO, L. DE F.; MARQUES JÚNIOR, J.; PEREIRA, G. T.; LA SCALA JUNIOR, N. Spatial variability of soil $\mathrm{CO}_{2}$ emission in different topography positions. Bragantia, v. 69, p. 19-27, 2010.

CAMARGO, L. A.; MARQUES JÚNIOR, J.; PEREIRA, G. T.; HORVAT, R. A. Variabilidade espacial de atributos mineralógicos de um Latossolo sob diferentes formas de relevo. I - Mineralogia da fração argila. Revista Brasileira de Ciência do Solo, v. 32, p. 2269-2277, 2008.

CORWIN, D. L.; LESCH, S. M. Apparent soil electrical conductivity measurements in agriculture. Computers \& Electronics in Agriculture, v. 46, p. 11-43, 2005.

EMBRAPA SOlOS. Manual de Métodos de Análise de Solo. 2.ed. rev. Rio de Janeiro: Embrapa Solos, 2011. (Documentos, 132) 
GÓES, M. H. B. Diagnóstico Ambiental por Geoprocessamento do Município de Itaguaí. 1994. 529 f. Tese (Doutorado em Geografia) - Instituto de Geociências e Ciências Exatas, Universidade Estadual Paulista Julio de Mesquita Filho, Rio Claro. 1994.

MANN, K. K.; SCHUMANN, A. W.; OBREZA, T. A.; HARRIS, W. G.; SHUKLA, S. Spatial variability of soil physical properties affecting Florida citrus production. Soil Science, v. 175, p. 487-499, 2010.

MELLO, G.; BUENO, C. R. P.; PEREIRA, G. T. Variabilidade espacial de perdas de solo, do potencial natural e risco de erosão em áreas intensamente cultivadas. Revista Brasileira de Engenharia Agrícola e Ambiental, v. 10, p. 315-322, 2006.

MELLO, L. C. Geofísica por eletrorresistividade e possibilidades de água subterrânea no assentamento rural Casas Altas-Eldorado, Seropédica, RJ. In: CONGRESSO BRASILEIRO DE ÁGUAS SUBTERRÂNEAS, 10., 1998, São Paulo. Anais... São Paulo: Associação Brasileira de Águas Subterrâneas, 1998.

MINASNY, B., MCBRATNEY, A. B. A conditioned Latin hypercube method for sampling in the presence of ancillary information. Computers \& Geosciences, v. 32, p. 1378-1388, 2006.

MONTANARI, R.; MARQUES JÚNIOR, J.; PEREIRA, G. T.; SOUZA, Z. M. Forma da paisagem como critério para otimização amostral de Latossolos sob cultivo de cana-de-açúcar. Pesquisa Agropecuária Brasileira, v. 40, p. 69-77, 2005.

PANOSSO, A. R.; MARQUES JÚNIOR, J.; PEREIRA, G. T.; LA SCALA JUNIOR, N. Spatial and temporal variability of soil $\mathrm{CO}_{2}$ emission in a sugarcane area under green and slash-and-burn managements. Soil \& Tillage Research, v. 105, p. 275-282, 2009.

PEBESMA, E. J. Multivariable geostatistics in S: the gstat package. Computers \& Geosciences, v. 30, p. 683-691, 2004.

R CORE TEAM. R: A Language and Environment for Statistical Computing. Viena, Áustria: R Foundation for Statistical Computing, 2015.

RODRIGUES, H. M.; VASQUES, G. M. Propriedades eletromagnéticas mostram potencial para mapear atributos do solo correlacionados em R. In: SEMINÁRIO INTERNACIONAL DE ESTATÍSTICA COM R, 1., 2016, Niterói. Anais... Niterói: Universidade Federal Fluminense, 2016.

RODRIGUES, H. M.; VASQUES, G. M.; COELHO, M. R. Sensores proximais como auxílio para tomadas de decisão em manejo do solo. In: REUNIÃO BRASILEIRA DE MANEJO E CONSERVAÇÃO DO SOLO E DA ÁGUA, 20., 2016, Foz do Iguaçu. Anais... Londrina: Instituto Agronômico do Paraná, 2016.

SANCHEZ, R. B.; MARQUES JÚNIOR, J.; PEREIRA, G. T.; SOUZA, Z. M. Variabilidade espacial de propriedades de Latossolo e da produção de café em diferentes superfícies geomórficas. Revista Brasileira de Engenharia Agrícola e Ambiental, v. 9, p. 489-495, 2005.

SILVA, A. M.; MELLO, C. R.; CURI, N. I.; OLIVEIRA, P. M. Simulação da variabilidade espacial da erosão hídrica em uma sub-bacia hidrográfica de Latossolos no sul de Minas Gerais. Revista Brasileira de Ciência do Solo, v. 32, p. 2125-2134, 2008.

WEBSTER, R.; OLIVER, M. A. Geostatistics for Environmental Scientists. 2.ed. Chichester: John Wiley \& Sons, 2007.

WILFORD, J. R., BIERWIRTH, P. N., CRAIG, M. A. Application of airborne gamma-ray spectrometry in soil/regolith mapping and applied geomorphology. AGSO Journal of Australian Geology and Geophysics, v. 17, p. 201-216, 1997. 